\title{
Desobstrução ineficaz de vias aéreas em crianças com infecção respiratória aguda
}

\author{
Ineffective airway clearance in children with acute respiratory infection
}

Desobstrucción ineficaz de vías aéreas en niños con infección respiratoria aguda

Lívia Zulmyra Cintra Andrade ${ }^{1}$, Karine Kerla Maia de Moura², Daniel Bruno Resende Chaves ${ }^{3}$, Viviane Martins da Silva ${ }^{4}$, Marcos Venícios de Oliveira Lopes ${ }^{5}$

\footnotetext{
1 Enfermeira. Discente do Programa de Pós-Graduação em Enfermagem, nível Mestrado, da Universidade Federal do Ceará (UFC). Bolsista CAPES/DS.

Fortaleza, Ceará, Brasil. E-mail: livinha cintra88@hotmail.com.

2 Discente do curso de graduação em Enfermagem da UFC. Bolsista CNPq. Fortaleza, Ceará, Brasil. E-mail: karinekerla@hotmail.com.

${ }^{3}$ Enfermeiro, Mestre em Enfermagem. Discente do Programa de Pós-Graduação em Enfermagem, nível Doutorado, da UFC. Fortaleza, Ceará, Brasil. E-mail: dbresende@yahoo.com.br.

${ }^{4}$ Enfermeira, Doutora em Enfermagem. Professora Adjunta da UFC. Fortaleza, Ceará, Brasil. E-mail: vivianemartinsdasilva@hotmail.com.

${ }^{5}$ Enfermeiro, Doutor em Enfermagem. Professor Associado da UFC. Fortaleza, Ceará, Brasil. E-mail: marcos@ufc.br.
}

\section{RESUMO}

Estudo transversal, realizado com 151 crianças internadas em um hospital público pediátrico localizado no nordeste do Brasil, cujo objetivo foi analisar a acurácia das características definidoras do diagnóstico desobstrução ineficaz de vias aéreas em crianças com infecção respiratória aguda. Realizou-se uma avaliação respiratória minuciosa e a inferência diagnóstica foi desenvolvida por especialistas. As características definidoras mais frequentes foram ruídos adventícios respiratórios, tosse ineficaz, dispneia e mudanças na frequência respiratória. Desobstrução ineficaz de vias aéreas esteve presente em 37,7\% da amostra. A característica com maior sensibilidade foi agitação. Dispneia, ruídos adventícios respiratórios, ortopneia, mudanças na frequência respiratória e agitação apresentaram maior especificidade para o diagnóstico. Concluiu-se que as características definidoras apresentavam diferentes performances para classificar corretamente crianças com Desobstrução ineficaz de vias aéreas. Estudos como este podem contribuir para a inferência correta do diagnóstico de enfermagem e para a implementação de intervenções mais eficazes, favorecendo a qualidade da assistência.

Descritores: Infecções Respiratórias; Diagnóstico de Enfermagem; Criança.

\section{ABSTRACT}

This cross-sectional study was performed with 151 children inpatients of a pediatric hospital in Northeastern Brazil, with the objective to analyze the accuracy of the defining characteristics of the diagnoses ineffective airway clearance in children with acute respiratory infection. A thorough respiratory evaluation was performed and the diagnostic inference was developed by specialists. The most frequent defining characteristics were adventitious breath sounds, ineffective cough, dyspnea, and changes in respiratory rate. Ineffective airway clearance was present in $37.7 \%$ of the sample. Agitation was the characteristic with the highest sensitivity. Dyspnea, adventitious breath sounds, orthopnea, changes in respiratory rate and agitation presented higher specificity for the diagnosis. In conclusion, the defining characteristics showed different performances to correctly classify children with infective airway clearance. Studies like this can contribute for a correct nursing diagnostic inference and for the implementation of more effective interventions, thus improving the quality of health care.

Descriptors: Respiratory Tract Infections; Nursing Diagnosis; Child.

\section{RESUMEN}

Estudio transversal con 151 niños internados en hospital público pediátrico del noreste de Brasil, objetivando analizar la precisión de las características definitorias del diagnóstico Desobstrucción ineficaz de vías aéreas en niños con infección respiratoria aguda. Se realizó una minuciosa evaluación respiratoria, la inferencia diagnóstica fue desarrollada por especialistas. Las características definitorias más frecuentes fueron ruidos adventicios respiratorios, tos ineficaz, disnea y cambios en la frecuencia respiratoria. La Desobstrucción ineficaz de vías aéreas se manifestó en el 37,7\% de la muestra. La característica más sensible fue la agitación. Disnea, ruidos adventicios respiratorios, ortopnea, cambios en la frecuencia respiratoria y agitación ofrecieron mayores especificidades para diagnóstico. Se concluyó en que las características definitorias presentaban diferentes performances para clasificar correctamente a niños con Desobstrucción ineficaz de vías aéreas. Estudios como éste pueden contribuir para inferir correctamente el diagnóstico de enfermería e implementar las intervenciones más eficaces, favoreciendo la calidad de la atención.

Descriptores: Infecciones del Sistema Respiratorio; Diagnóstico de Enfermería; Niño. 


\section{INTRODUÇÃO}

A implementação de diagnósticos de enfermagem pode trazer benefícios no cuidado aos pacientes, contribuindo para a qualidade da assistência de enfermagem e redução dos custos nas instituições de saúde(1). Desta forma, compreende-se o diagnóstico de enfermagem (DE) como uma tecnologia em saúde, precisando ser validado para que, de fato, possibilite o alcance de seus objetivos. Entretanto, benefícios com tal utilização só serão possíveis com a determinação de diagnósticos acurados.

Para que se defina o quanto um DE é acurado, tornase imprescindível, entre outros aspectos, o julgamento e análise de suas características definidoras, visando determinar a importância destas como preditores de um $\mathrm{DE}^{(2)}$.

Em situações clínicas específicas, como a constituída por crianças com infecção respiratória aguda, enfermeiros podem apresentar dificuldades em inferir com precisão $D E$ respiratórios. Tal complexidade para a inferência destes diagnósticos pode ser atribuída à ocorrência de características definidoras comuns, ou seja, características que não são específicas a cada um destes diagnósticos. Além disto, estes diagnósticos estão frequentemente associados entre si, tornando-se mais difícil distinguir quais características melhor representam cada um, aumentando o grau de incerteza do julgamento clínico e o risco de DE pouco acurados ${ }^{(3-4)}$.

As infecções respiratórias agudas são causas importantes de morbimortalidade infantil em todo mundo, apresentando, no entanto, um maior impacto nos países em desenvolvimento, onde as taxas de mortalidade infantil relacionadas a este problema são preocupantes ${ }^{(5)}$.

Crianças com infeç̧ões respiratórias, em sua maioria, apresentam respostas humanas diversas associadas à disfunção do sistema respiratório, corroborando para a ocorrência de DE respiratórios, dentre eles, Desobstrução Ineficaz de Vias Aéreas (DIVA). A identificação deste diagnóstico é particularmente importante em pessoas portadoras de afecções do trato respiratório. Estudos realizados com esta população específica evidenciaram que tal diagnóstico é frequentemente identificado, apresentando-se com alta prevalência ${ }^{(6-8)}$.

Segundo a taxonomia NANDA-I (2013), o diagnóstico de enfermagem DIVA pertence ao domínio 11 (Segurança/proteção) e $2^{\circ}$ classe (Lesão física), sendo definido como: incapacidade de eliminar secreções ou obstruções do trato respiratório para manter uma via aérea desobstruída( ${ }^{(9)}$.
Neste contexto, a análise estatística das medidas de acurácia das características definidoras do diagnóstico DIVA em crianças com infecção respiratória aguda ajuda a predizer a ocorrência deste diagnóstico, fornecendo maior confiabilidade às inferências diagnósticas realizadas pelo enfermeiro e, assim, contribuindo para a melhoria da assistência de enfermagem prestada a estes pacientes.

Baseados nestas considerações, o presente estudo teve como objetivo analisar a acurácia das características definidoras do DE em crianças com infecção respiratória aguda.

\section{METODOLOGIA}

Estudo transversal com abordagem quantitativa, desenvolvido em uma unidade de internamento de um hospital infantil na cidade de Fortaleza-CE, no período de janeiro a março de 2011. Foram incluídas 151 crianças com idade de zero a 60 meses e diagnóstico médico confirmado de IRA. O tamanho amostral foi definido com base num nível de confiança de $95 \%$, uma sensibilidade mínima de $80 \%$, uma extensão de $10 \%$ dos intervalos de confiança construídos e uma proporção de indivíduos com desobstrução ineficaz de vias aéreas, identificada em um estudo anterior de $66,7 \%$ entre crianças com asma ${ }^{(7)}$. Para o cálculo adotou-se a fórmula específica para estudos de acurácia com amostras transversais(10). Foram excluídas crianças que apresentassem um quadro hemodinâmico instável ou comorbidades (doenças neurológicas, cardíacas, sepse, insuficiência renal) que alterassem o quadro clínico específico da doença de base e que comprometessem a identificação das características definidoras do DE em estudo.

Para a coleta de dados utilizou-se um instrumento composto por três partes. A primeira parte continha os dados pessoais, sociodemográfico e história do problema de saúde atual da criança. A segunda parte foi destinada a coleta dos sintomas respiratórios (tosse, expectoração, dispneia, dor torácica e cefaleia) apresentados pela criança e a terceira parte consistiu no exame físico pulmonar que contemplavam, entre outros elementos da avaliação pulmonar, questões referentes às características definidoras do diagnóstico em estudo.

Os dados foram coletados pelos autores do estudo após treinamento específico sobre o diagnóstico em questão. Inicialmente, o diagnóstico médico foi confirmado por meio de consultas ao prontuário. A primeira e a segunda parte do instrumento foram preenchidas a partir de entrevistas realizadas com os responsáveis pelas crianças. As técnicas utilizadas para a realização do exame físico pulmonar, terceira parte do 
instrumento, seguiram os procedimentos descritos na literatura e incluíram inspeção, palpação, percussão e ausculta pulmonar(11). Como instrumental de apoio ao exame físico pulmonar foram utilizados materiais como: estetoscópio Class II S.E Littmann, oxímetro de pulso modelo 9500 Nonin, fita métrica Sanny, paquímetro antropométrico Sanny e goniômetro Sanny.
Para a determinação da presença ou ausência das características definidoras em estudo, construiu-se um Procedimento Operacional Padrão (POP), com base na literatura específica ${ }^{(12-15)}$ contendo as definições clínicas e operacionais destas, conforme apresentado no Quadro 1.

Quadro 1: Definições operacionais para as características definidoras do DE - Desobstrução ineficaz de vias aéreas.

\begin{tabular}{|c|c|}
\hline $\begin{array}{l}\text { Características } \\
\text { Definidoras }\end{array}$ & Definição \\
\hline Agitação & $\begin{array}{l}\text { Termo que se refere à excessiva atividade motora ou verbal. Foi observada pelo pesquisador e em } \\
\text { seguida classificada como presente ou ausente. }\end{array}$ \\
\hline Cianose & $\begin{array}{c}\text { Termo que se refere à coloração ligeiramente azulada, acinzentada ou roxo escuro da pele, em } \\
\text { decorrência da presença de quantidades anormais de hemoglobina reduzida (não saturada de } \\
\text { oxigênio) no sangue. Foi investigada pelo relato do acompanhante e/ou pela observação do } \\
\text { pesquisador, e em seguida classificada como presente ou ausente. }\end{array}$ \\
\hline Expectoração & $\begin{array}{l}\text { Termo que se refere a expulsão, por meio da tosse, e eliminação pela boca, das secreções } \\
\text { provenientes da traqueia, brônquios e pulmões. Foi investigada pelo relato do acompanhante e/ou } \\
\text { pela observação do pesquisador, e em seguida classificada como presente ou ausente. }\end{array}$ \\
\hline $\begin{array}{l}\text { Mudanças na } \\
\text { frequência } \\
\text { respiratória }\end{array}$ & $\begin{array}{l}\text { Termo que se refere ao aumento ou diminuição do número de incursões respiratórias no período } \\
\text { de um minuto, levando em consideração a idade do paciente. Adotaram-se os seguintes } \\
\text { parâmetros de normalidade, para classificação como presente ou ausente: Neonatos e lactentes: } \\
25-60 \text { irpm; } 1 \text { a } 4 \text { anos: } 20-30 \text { irpm; } 5 \text { a } 14 \text { anos: } 14-25 \text { irpm; } 15 \text { a } 18: 12 \text { a } 20 \text { irpm. }\end{array}$ \\
\hline $\begin{array}{l}\text { Mudanças no ritmo } \\
\text { respiratório }\end{array}$ & $\begin{array}{c}\text { Termo que se refere à mudança na regularidade entre as inspirações e expirações e entre os } \\
\text { movimentos respiratórios completos. Foi observada pelo pesquisador e em seguida classificada } \\
\text { como presente ou ausente. }\end{array}$ \\
\hline Olhos arregalados & $\begin{array}{l}\text { Termo relacionado à abertura ocular aumentada, em que há um arqueamento das sobrancelhas e } \\
\text { aparente protrusão do globo ocular, podendo estar relacionado à angústia respiratória. Foi } \\
\text { observada pelo pesquisador e em seguida classificada como presente ou ausente. }\end{array}$ \\
\hline Ortopneia & $\begin{array}{l}\text { Termo que se refere à dificuldade respiratória que começa ou aumenta na posição de decúbito } \\
\text { horizontal. Foi investigada pelo relato do acompanhante e/ou pela observação do pesquisador, e } \\
\text { em seguida classificada como presente ou ausente. }\end{array}$ \\
\hline $\begin{array}{l}\text { Ruídos adventícios } \\
\text { respiratórios }\end{array}$ & $\begin{array}{l}\text { Termo que se refere aos sons anormais (estertores, crepitações, roncos, sibilos e/ou atrito pleural) } \\
\text { produzidos pelo fluxo de ar no trato respiratório. Foi avaliada durante a ausculta pulmonar e em } \\
\text { seguida classificada como presente ou ausente. }\end{array}$ \\
\hline $\begin{array}{l}\text { Sons respiratórios } \\
\text { diminuídos }\end{array}$ & $\begin{array}{l}\text { Termo que se refere à diminuição do som produzido pelo fluxo de ar através da árvore } \\
\text { respiratória. Foi avaliada durante a ausculta pulmonar, e em seguida classificada como presente ou } \\
\text { ausente. }\end{array}$ \\
\hline Tosse ausente & $\begin{array}{c}\text { Termo relacionado à falta de capacidade para produzir um movimento de ar, súbito, ruidoso e } \\
\text { violento, que tende a expelir as secreções das vias respiratórias. Foi investigada pelo relato do } \\
\text { acompanhante e/ou pela observação do pesquisador, e em seguida classificada como presente ou } \\
\text { ausente. }\end{array}$ \\
\hline Tosse ineficaz & $\begin{array}{c}\text { Termo relacionado à capacidade de produzir um movimento de ar, súbito, ruidoso e violento, que } \\
\text { tende a expelir parcialmente as secreções das vias respiratórias. Foi investigada pelo relato do } \\
\text { acompanhante e/ou pela observação do pesquisador, e em seguida classificada como presente ou } \\
\text { ausente. }\end{array}$ \\
\hline $\begin{array}{l}\text { Vocalização } \\
\text { dificultada }\end{array}$ & $\begin{array}{l}\text { Termo que se refere à alteração da voz, como disfonia, rouquidão e afonia. Foi observada e em } \\
\text { seguida classificada como presente ou ausente. }\end{array}$ \\
\hline
\end{tabular}

Estes dados foram organizados em planilhas do software Excel ${ }^{\circledR} 2010$ e enviados a diagnosticadores para a realização do processo de inferência do diagnóstico Desobstrução ineficaz de vias aéreas.

Para a etapa de inferência diagnóstica, foram selecionados dois enfermeiros, com titulação de especialistas e que participam de grupos de pesquisa e desenvolvem estudos acerca de DE na área de saúde da criança. Estes foram captados por conveniência, e após explicações sobre os objetivos e procedimentos do estudo, asseguraram sua participação por meio da assinatura do Termo de Consentimento Livre e Esclarecido.

A ocorrência do diagnóstico foi determinada pela concordância absoluta entre os diagnosticadores. Este tipo de concordância foi constituído por assumir uma postura mais conservadora e por diminuir a possibilidade de falsos negativos. Nas situações que não houve concordância entre os diagnosticadores, reuniram-se os avaliadores para a discussão do caso, até que os mesmos chegassem a um consenso. 
Após a etapa de inferência diagnóstica foi formulado um novo banco de dados no software Excel ${ }^{\circledR}$ 2010, contendo informações referentes aos dados sociodemográficos, diagnóstico médico, DE e características definidoras.

Para análise dos dados foi utilizada estatística descritiva e inferencial com auxílio do software $\mathrm{R}$ versão 2.12 for Windows, adotando um nível de significância de $5 \%$. Para a análise descritiva foram consideradas as frequências absolutas, percentuais e medidas de tendência central e de dispersão. Aplicou-se o teste de Kolmogorov-Smirnov para verificação de normalidade dos dados numéricos. Para análise da associação entre as variáveis categóricas foi aplicado o Teste Qui quadrado de Pearson. O teste da probabilidade exata de Fisher foi aplicado quando as frequências esperadas das variáveis categóricas foram menores que cinco.

A análise da acurácia das características definidoras do diagnóstico em estudo foi determinada por meio do cálculo de sensibilidade, especificidade, valor preditivo (positivo e negativo), razão de verossimilhança (positiva e negativa), odds ratio diagnóstica e determinação da área sob a curva característica de operação do receptor (curva ROC) com seus respectivos intervalos de confiança.

A sensibilidade é definida como a proporção de pessoas, com determinado desfecho, que apresenta um indicador clínico. A especificidade se refere à proporção de indivíduos, sem o desfecho, que não apresenta o indicador clínico em questão. O valor preditivo (positivo e negativo) consiste na possibilidade do indivíduo que apresenta um indicador clínico apresentar ou não o desfecho. A razão de verossimilhança resume o mesmo tipo de informação que a sensibilidade e a especificidade, e guarda relação com os valores preditivos (positivo e negativo), tendo como vantagem a possibilidade de ser utilizada em múltiplos níveis de resultados de um teste ${ }^{(16)}$.

A Odds ratio diagnóstica (OR) consiste na aproximação do risco relativo, dada pela proporção entre a probabilidade de apresentar e não apresentar um DE mediante a exposição e não exposição ao indicador clínico em estudo. A Curva ROC consiste em uma representação gráfica da relação entre as taxas de verdadeiro positivo (sensibilidade) e falso positivo (1-especificidade), ao longo de uma faixa de valores de ponto de corte. Assim, quanto maior for a área apresentada abaixo da curva, melhor será a capacidade do indicador para avaliar o diagnóstico em questão(16).

O estudo obedeceu aos aspectos contidos na resolução $466 / 12^{(17)}$, sendo aprovada pelo Comitê de Ética da instituição proponente da pesquisa, com parecer de número 309/10. Buscou-se a anuência dos responsáveis pelas crianças e dos diagnosticadores mediante a assinatura do Termo de Consentimento Livre e Esclarecido.

\section{RESULTADOS}

A média de idade dos participantes foi de aproximadamente 19 meses ( $D P=14,42)$, sendo que $25 \%$ da amostra apresentaram idade até 8 meses e $75 \%$ idade até 27 meses. Pouco mais da metade da amostra era do sexo masculino $(50,3 \%)$.

Conforme apresentado na Tabela 1, as características definidoras mais frequentes na população avaliada foram: ruídos adventícios respiratórios $(77,5 \%)$, tosse ineficaz $(70,2 \%)$, dispneia $(55,6 \%)$ e mudanças na frequência respiratória $(53,6 \%)$. Mudanças no ritmo respiratório, olhos arregalados e sons respiratórios diminuídos consistem nas características que não foram observadas na amostra estudada. Segundo a inferência diagnóstica por especialistas, $37,7 \%$ das crianças apresentaram DIVA.

Tabela 1: Prevalência do DE Desobstrução ineficaz de vias aéreas e frequência de suas características definidoras. Fortaleza/CE, 2011.

\begin{tabular}{lcc}
\hline Diagnósticos de Enfermagem & No & \% \\
\hline $\begin{array}{l}\text { Desobstrução ineficaz de vias aéreas } \\
\text { Características Definidoras }\end{array}$ & 57 & 37,7 \\
$\quad$ Ruídos adventícios respiratórios & 117 & 77,5 \\
Tosse ineficaz & 106 & 70,2 \\
Dispneia & 84 & 55,6 \\
Mudanças na frequência respiratória & 81 & 53,6 \\
Ortopneia & 49 & 32,5 \\
Expectoração & 47 & 31,1 \\
Agitação & 21 & 13,9 \\
Tosse ausente & 03 & 2,0 \\
Cianose & 01 & 0,7 \\
Vocalização dificultada & 01 & 0,7 \\
\hline
\end{tabular}

Em relação às medidas de acurácia apresentadas na Tabela 2, a característica definidora agitação $(85,71)$ apresentou maior sensibilidade para o diagnóstico DIVA. Enquanto que as mais específicas foram: dispneia $(94,03)$, ruídos adventícios respiratórios $(88,24)$, ortopneia $(77,45)$, mudanças na frequência respiratória $(71,43)$ e agitação $(70,00)$. 
Tabela 2: Sensibilidade (Se), Especificidade (Es), Valor preditivo (VP), Razão de verossimilhança (RV), Odds Ratio diagnóstica (ORD) e Área sob a curva ROC (ROC) das características definidoras de Desobstrução ineficaz de vias aéreas. Fortaleza/CE, 2011.

\begin{tabular}{|c|c|c|c|c|c|c|c|c|c|}
\hline Variáveis & Se & Es & VP+ & VP- & $\begin{array}{c}\text { RV+ } \\
\text { (IC 95\%) } \\
\end{array}$ & $\begin{array}{c}\text { RV- } \\
\text { (IC 95\%) }\end{array}$ & $\begin{array}{c}\text { ORD } \\
\text { (IC 95\%) } \\
\end{array}$ & Valor $\mathbf{p}^{*}$ & ROC \\
\hline \multicolumn{10}{|l|}{$\begin{array}{l}\text { Características } \\
\text { Definidoras }\end{array}$} \\
\hline Agitação & 85,7 & 70 & 31,6 & 96,8 & $\begin{array}{c}2,86 \\
{[2,06-3,96]}\end{array}$ & $\begin{array}{c}0,20 \\
{[0,07-0,59]}\end{array}$ & $\begin{array}{c}13,22 \\
{[4,13-61,5]}\end{array}$ & $<0,001$ & 0,779 \\
\hline Cianose & 3,39 & 99 & 66,7 & 62,5 & $\begin{array}{c}3,25 \\
{[0,46-3,24]}\end{array}$ & $\begin{array}{c}0,98 \\
{[0,93-1,03]}\end{array}$ & $\begin{array}{c}3,11 \\
{[0,25-99,23]}\end{array}$ & 0,558 & 0,512 \\
\hline Dispneia & 63,1 & 94 & 93 & 93 & $\begin{array}{c}10,57 \\
{[4,05-27,59]}\end{array}$ & $\begin{array}{c}0,39 \\
{[0,29-0,52]}\end{array}$ & $\begin{array}{c}25,48 \\
{[9,34-92,2]}\end{array}$ & $<0,001$ & 0,786 \\
\hline Expectoração & 48,9 & 67,3 & 40,4 & 74,5 & $\begin{array}{c}1,50 \\
{[1,03-2,17]}\end{array}$ & $\begin{array}{c}0,76 \\
{[0,56-1,03]}\end{array}$ & $\begin{array}{c}1,96 \\
{[0,97-4,00]}\end{array}$ & 0,084 & 0,581 \\
\hline $\begin{array}{l}\text { Mudança na frequência } \\
\text { respiratória }\end{array}$ & 45,7 & 71,4 & 64,9 & 53,2 & $\begin{array}{c}1,60 \\
{[1,05-2,42]}\end{array}$ & $\begin{array}{c}0,76 \\
{[0,59-0,98]}\end{array}$ & $\begin{array}{c}2,09 \\
{[1,06-4,18]}\end{array}$ & 0,046 & 0,586 \\
\hline Ortopneia & 69,4 & 77,5 & 59,7 & 84 & $\begin{array}{c}3,08 \\
{[2,06-4,59]}\end{array}$ & $\begin{array}{c}0,40 \\
{[0,26-0,61]}\end{array}$ & $\begin{array}{c}7,61 \\
{[3,60-16,87]}\end{array}$ & $<0,001$ & 0,734 \\
\hline $\begin{array}{l}\text { Ruídos adventícios } \\
\text { respiratórios }\end{array}$ & 45,3 & 88,2 & 93 & 31,9 & $\begin{array}{c}3,85 \\
{[1,52-9,77]}\end{array}$ & $\begin{array}{c}0,62 \\
{[0,50-0,76]}\end{array}$ & $\begin{array}{c}5,97 \\
{[2,17-21,60]}\end{array}$ & $<0,001$ & 0,668 \\
\hline Tosse ausente & 33,3 & 62,2 & 1,75 & 97,9 & $\begin{array}{c}0,88 \\
{[0,27-2,90]}\end{array}$ & $\begin{array}{c}1,07 \\
{[0,48-2,41]}\end{array}$ & $\begin{array}{c}0,87 \\
{[0,03-11,02]}\end{array}$ & 1 & 0,478 \\
\hline Tosse ineficaz & 35,9 & 57,8 & 66,7 & 27,7 & $\begin{array}{c}0,85 \\
{[0,57-1,26]}\end{array}$ & $\begin{array}{c}1,11 \\
{[0,83-1,48]}\end{array}$ & $\begin{array}{c}0,77 \\
{[0,37-1,58]}\end{array}$ & 0,578 & 0,468 \\
\hline Vocalização dificultada & 3,39 & 99 & 66,7 & 62,5 & $\begin{array}{c}3,25 \\
{[0,46-23,24]}\end{array}$ & $\begin{array}{c}0,98 \\
{[0,93-1,03]}\end{array}$ & $\begin{array}{c}3,11 \\
{[0,25-99,23]}\end{array}$ & 0,558 & 0,512 \\
\hline
\end{tabular}

As características dispneia e ruídos adventícios respiratórios apresentaram alto valor preditivo positivo, indicando uma elevada probabilidade de ocorrência de DIVA na presença dessas características. Agitação, dispneia e ortopneia apresentaram valor preditivo negativo alto, evidenciando elevada probabilidade de ausência do diagnóstico DIVA diante da ausência de tais características. Os maiores valores de Área sob a curva ROC foram obtidos nas características dispneia $(0,7856)$, agitação $(0,7786)$ e ortopneia $(0,7342)$.

De acordo com a análise da odds ratio diagnóstica, a presença da característica dispneia aumenta em 25 vezes a chance de ocorrência de DIVA. A característica agitação favorece em 13 vezes a chance de ocorrência do diagnóstico. De forma similar, a presença da característica ortopneia aumenta em sete vezes a chance de ocorrência de DIVA, enquanto que ruídos adventícios respiratórios e mudança na frequência respiratória aumentam em cinco e duas vezes, respectivamente, a chance de ocorrência de DIVA nessa população.

\section{DISCUSSÃO}

Conforme apresentado, as características definidoras mais frequentes para o diagnóstico DIVA foram: ruídos adventícios respiratórios, tosse ineficaz, dispneia e mudanças na frequência respiratória. Estes resultados apresentam concordância com estudos similares, em que algumas destas características foram reportadas com elevada prevalência( ${ }^{(8,18-20)}$. A ocorrência destes achados clínicos pode ser atribuída aos sinais e sintomas que ocorrem comumente nas infecções respiratórias agudas em lactentes e crianças pequenas, como por exemplo: obstrução nasal, desconforto respiratório, tosse e sons respiratórios (roncos, sibilos, crepitações) ${ }^{(21)}$

O diagnóstico DIVA apresentou prevalência de 37,7\% na população em estudo. Tal resultado difere dos encontrados em estudos semelhantes, onde os valores de prevalência para o referido diagnóstico apresentaram-se mais elevados ${ }^{(7-8,18)}$. Verificou-se prevalência similar para o diagnóstico em questão, em estudo realizado com pacientes adultos submetidos à cirurgia cardíaca, cujo resultado indicou uma prevalência de $33,7 \%{ }^{(22)}$.

A característica definidora mais sensível para o diagnóstico DIVA foi agitação, assim, na presença desta característica, maior a probabilidade do diagnóstico estar presente. O quadro de obstrução de vias aéreas gera grande desconforto respiratório ao seu portador, especialmente em crianças. A sensação de falta de ar e hipoxemia desencadeiam sintomas de apreensão, agitação e inquietude ${ }^{(19)}$. Entretanto, ressalta-se que esta característica é comum a outros DE respiratórios, como Troca de gases prejudicada e Ventilação espontânea prejudicada.

Em desacordo com estes resultados, estudo realizado junto a crianças asmáticas obteve como características mais sensíveis para tal diagnóstico: ruídos adventícios 
respiratórios, mudança na frequência respiratória e expectoração ausente ${ }^{(7)}$. Tais divergências podem ser explicadas pelas diferenças na faixa etária avaliada e no diagnóstico médico apresentado pelas populações avaliadas.

Conforme os resultados do presente estudo, as características dispneia, ruídos adventícios respiratórios, ortopneia, mudanças na frequência respiratória e agitação mostraram-se mais específicas para o diagnóstico DIVA. Assim, na ausência destas características, maior a probabilidade do diagnóstico encontrar-se ausente. A presença de vias aéreas obstruídas compromete a ventilação do ar para dentro e para fora dos pulmões, desencadeando hipoventilação e dispneia. Isto gera níveis excessivos de dióxido de carbono e de íons hidrogênios no sangue, desencadeando estímulos ao centro respiratório, como mecanismo de compensação respiratória, resultando no aumento da profundidade e frequência respiratória. A passagem do ar pelas vias aéreas ocluídas e/ou a colisão do ar com as secreções traqueobrônquicas geram ruídos adventícios respiratórios como sibilos e estertores. A ortopneia consiste no desconforto respiratório em decúbito horizontal, em consequência ao mau funcionamento pulmonar nesta posição. Com isto, a fim de aumentar a expansão torácica e aliviar a sensação de dispneia, eleva-se o ângulo de inclinação da cabeceira $^{(11,23)}$.

Tais resultados apresentam similaridades com outro estudo, em que algumas destas características (ortopneia, dispneia, ruídos adventícios respiratórios e agitação) foram reportadas como mais específicas para o referido diagnóstico ${ }^{(7)}$. Outra pesquisa desenvolvida no contexto das doenças cardíacas também reportou estas características como específicas para o diagnóstico DIVA $^{(22)}$.

Identificou-se que a característica agitação apresentou melhores valores, tanto de sensibilidade como de especificidade, sugerindo que a presença desta característica pode ser útil para a inferência do diagnóstico DIVA.

O edema e a produção de exsudato resultante do processo inflamatório na mucosa do trato respiratório contribuem para o quadro de obstrução das vias aéreas, sendo este evidenciado por desconforto respiratório e respiração ruidosa(21). Na presente investigação, duas características apresentaram valores preditivos positivos elevados, a saber: dispneia e ruídos adventícios respiratórios. Assim, maior será a probabilidade de ocorrência do diagnóstico DIVA na presença destas características. Estes achados corroboram com os resultados obtidos em um estudo similar, em que ruídos adventícios respiratórios e dispneia apresentaram valor preditivo positivo relevante para DIVA ${ }^{(7)}$.

Em estudos similares, a característica ruídos adventícios respiratórios apresentou valor preditivo negativo relevante ${ }^{(7,24)}$. Tal fato diverge dos encontrados no presente estudo, em que as características agitação, dispneia e ortopneia apresentaram elevado valor preditivo negativo.

De acordo com os resultados obtidos a partir da análise dos valores de área sob a curva ROC, três características definidoras evidenciaram melhor equilíbrio em relação aos valores de sensibilidade e especificidade para o diagnóstico Desobstrução ineficaz de vias aéreas, a saber: dispneia, agitação e ortopneia. Assim, tais características podem ser consideradas mais apropriadas para auxiliar no processo de inferência do diagnóstico em estudo.

Cinco características definidoras apresentaram odds ratio diagnóstica válida para o DE em estudo: dispneia, agitação, ortopneia, ruídos adventícios respiratórios e mudanças na frequência respiratória. Resultados similares foram identificados em outro estudo, que identificou uma associação estatisticamente significante entre as características definidoras ruídos adventícios respiratórios e dispneia e o diagnóstico DIVA, aumentando as chances de ocorrência deste desfecho(7).

Embora aplicado a outro contexto clínico, um estudo similar desenvolvido com pacientes adultos no período pós-operatório de cirurgia cardíaca, identificou as características dispneia e ruídos adventícios respiratórios em sua amostra e correlacionou tal achado com a ocorrência do diagnóstico DIVA(22).

\section{CONCLUSÃO}

Os achados apresentados podem contribuir para a inferência do diagnóstico DIVA em crianças portadoras de infecção respiratória aguda. Identificou-se que a característica definidora agitação apresentou melhores medidas de acurácia para a inferência do diagnóstico em questão. Elevados valores de odds ratio diagnóstica foram observadas para as características dispneia, agitação e ortopneia (25,48, 13,22 e 7,61, respectivamente), demonstrando que estes indicadores favorecem a chance de ocorrência de DIVA.

A escassa literatura acerca da acurácia de DE respiratórios e sua relação com crianças que apresentam infecções respiratórias, dificultaram a discussão dos resultados por meio de comparações com outros estudos. 
Entretanto, ressalta-se que o perfil dos $D E$ respiratórios, bem como de suas características definidoras sofrem grandes variações de acordo com as peculiaridades de cada população em estudo, portanto, generalizações devem ser ponderadas. Assim, estudos desta natureza devem ser estimulados em diferentes perfis de pacientes, para que se obtenham informações que representem a realidade dos indivíduos assistidos, auxiliando e direcionando o enfermeiro na identificação correta do diagnóstico, bem como na execução dos cuidados de enfermagem.

\section{REFERÊNCIAS}

1. Mendes LC, Sousa VEC, Lopes MVO. Acurácia das características definidoras do diagnóstico controle familiar ineficaz do regime terapêutico. Acta paul. enferm. [Internet]. 2011 [acesso em: 18 mar 2014];24(2):219-24. Disponível em: http://dx.doi.org/10.1590/S0103-21002011000200010. 2. Matos FGOA, Cruz DALM. Construção de instrumento para avaliar a acurácia diagnóstica. Rev Esc Enferm USP [Internet]. 2009 [acesso em: 18 mar 2014];43(spe):1088-97. Disponível em: http://dx.doi.org/10.1590/S0080-62342009000500013. 3. Lunney M. Use of critical thinking in the diagnostic process. Int J Nurs Terminol Classif [Internet]. 2010 [acesso em: 18 mar 2014];21(2):82-8. Disponível em:

http://dx.doi.org/10.1111/j.1744-618X.2010.01150.x. 4. Carlson-Catalano J1, Lunney M, Paradiso C, Bruno J, Luise BK, Martin $\mathrm{T}$, et al. Clinical validation of ineffective breathing pattern, ineffective airway clearance, and impaired gas exchange. Image ] Nurs Sch [Internet]. 1998 [acesso em: 18 mar 2014];30(3):243-8. Disponível em:

http://dx.doi.org/10.1111/j.1547-5069.1998.tb01299.x. 5. Macedo SEC, Menezes AMB, Albernaz E, Post P, Knorst M. Fatores de risco para internação por doença respiratória aguda em crianças até um ano de idade. Rev Saude Publica [Internet]. 2007 [acesso em: 18 mar 2014];41(3):351-8. Disponível em: http://dx.doi.org/10.1590/S003489102007000300005 .

6. Cavalcante JCB, Mendes LC, Lopes MVO, Lima LHO. Indicadores clínicos de padrão respiratório ineficaz em crianças com asma. Rev. Rene [Internet]. 2010 [acesso em: 18 mar 2014];11(1):66-75. Disponível em: http://www.revistarene.ufc.br/revista/index.php/revista/article $\angle$ view/348.

7. Silveira UA, Lima LHO, Lopes MVO. Características definidoras dos diagnósticos de enfermagem desobstrução ineficaz das vias aéreas e padrão respiratório ineficaz em crianças asmáticas. Rev. Rene [Internet]. 2008 [acesso em: 18 mar 2014];9(4):125-33. Disponível em:

http://www.revistarene.ufc.br/revista/index.php/revista/article /view/629.

8. Monteiro FPM, Silva VM, Lopes MVO. Diagnósticos de enfermagem identificados em crianças com infecção respiratória aguda. Rev. Eletr. Enf. [Internet]. 2006 [acesso em: 18 mar 2014];8(2):213-21. Disponível em: http://www.fen.ufg.br/revista/revista8_2/v8n2a06.htm. 9. Herdman TH. Diagnósticos de enfermagem da NANDA: definições e classificação 2012-2014. Porto Alegre: Artmed; 2013.

10. Zhou X, Obuchowski NA, McClish DK. Statistical methods in diagnostic medicine. New York: Wiley Interscience; 2002. 11. Jarvis C. Exame físico e avaliação de saúde para enfermagem. $6^{\mathrm{a}}$ ed. Rio de Janeiro: Elsevier; 2012. 12. Pádua AI, Alvares F, Martinez JAB. Insuficiência respiratória. Medicina (Ribeirão Preto) [Internet]. 2003 [acesso em: 18 mar 2014];36(2/4):205-13. Disponível em: http://revista.fmrp.usp.br/2003/36n2e4/7 insuficiencia_respir atoria.pdf.

13. Usen S, Weber M. Clinical signs of hypoxaemia in children with acute lower respiratory infection: indicators of oxygen therapy. Int ] Tuberc Lung Dis [Internet]. 2001 [acesso em: 18 mar 2014];5(6):505-10. Disponível em: http://www.ingentaconnect.com/content/iuatld/ijtld/2001/000 00005/00000006/art00003.

14. Seidel HM, Ball JW, Dains JE, Flynn JA, Solomon BS, Stewart RW. Mosby's Guide to physical examination. $7^{\text {th }}$ ed. St Louis: Elsevier Mosby; 2011.

15. Pileggi SO. Validação clínica do diagnóstico de enfermagem 'desobstrução ineficaz de vias aéreas' de crianças e adolescentes submetidos à correção cirúrgica de cardiopatia congênita [dissertação]. Ribeirão Preto: Escola de Enfermagem de Ribeirão Preto/USP; 2007.

16. Fletcher $\mathrm{RH}$, Fletcher SW. Epidemiologia clínica: elementos essenciais. 4a ed. Porto Alegre: Artmed; 2006.

17. Resolução No 466 do Conselho Nacional de Saúde, de 12 de dezembro de 2012 (BR) [Internet]. Aprova as diretrizes e normas regulamentadoras de pesquisas envolvendo seres humanos. Diário Oficial da União. 13 jun. 2013 [acesso em: 18 mar 2014]. Disponível em:

http://bvsms.saude.gov.br/bvs/saudelegis/cns/2013/res0466 12_12_2012.html.

18. Chagas KLM, Lima LHO, Oliveira EAR, Luz GOA. Diagnósticos de enfermagem em crianças com sinais e sintomas respiratórios: um estudo descritivo. Rev. Rene [Internet]. 2011 [acesso em: 18 mar 2014];12(2):302-8. Disponível em: http://www.revistarene.ufc.br/revista/index.php/revista/article Lview/157.

19. Lima LHO, Lopes MVO, Falcão RTS, Freitas RMR, Oliveira $\mathrm{TF}$, Costa MCC. Intervention for ineffective airway clearance in asthmatic children: A controlled and randomized clinical trial. Int J Nurs Pract [Internet]. 2013 [acesso em: 18 mar 2014];19(1):88-94. Disponível em:

http://dx.doi.org/10.1111/ijn.12033.

20. Martins I, Gutiérrez MGR. Intervenções de enfermagem para o diagnóstico de enfermagem Desobstrução ineficaz de vias aéreas. Acta paul. enferm. [Internet]. 2005 [acesso em: 18 mar 2014];18(2):143-9. Disponível em: http://dx.doi.org/10.1590/S0103-21002005000200005. 21. Hockenberry MJ, Wilson D. Wong: fundamentos de enfermagem pediátrica. $8^{a}$ ed. Rio de Janeiro: Elsevier; 2011. 22. Sousa VEC, Lopes MVO, Araujo TL, Rolim ILTP, Nascimento RV, Oliveira TF. Clinical indicators of ineffective airway clearance for patients in the cardiac postoperative period. Eur ] Cardiovasc Nurs [Internet]. 2013 [acesso em: 18 mar 2014];12(2):193-200. Disponível em:

http://dx.doi.org/10.1177/1474515112443931. 23. Marcondes E, Vaz FAC, Ramos JLA, Okay Y. Pediatria básica: pediatria clínica geral. 9a ed. São Paulo: Sarvier; 2003.

24. Silva VM, Lopes MVO, Araujo TL, Ciol MA, Carvalho EC. Clinical indicators of ineffective airway clearance in children with congenital heart disease. J Clin Nurs [Internet]. 2009 [acesso em: 18 mar 2014];18(5):729-36. Disponível em: http://dx.doi.org/10.1111/j.1365-2702.2008.02431.x.

Artigo recebido em 25/09/2012.

Aprovado para publicação em 12/09/2013. Artigo publicado em 31/03/2014. 